\title{
Patrones de distribución espacial de ensambles de macroinvertebrados bentónicos de un sistema fluvial Andino Patagónico
}

\author{
Spatial distribution patterns of benthic macroinvertebrates assemblages in an Andean \\ Patagonian fluvial system
}

\begin{abstract}
CAROLINA MOYA ${ }^{1, *}$, CLAUDIO VALDOVINOS ${ }^{1,2}$, ANDRÉS MORAGA ${ }^{1}$, FRANCISCO ROMERO ${ }^{3}$, PATRICK DEBELS ${ }^{1,4} \&$ ALEJANDRA OYANEDEL ${ }^{1}$
\end{abstract}

\author{
${ }^{1}$ Unidad de sistemas acuáticos, Centro Universitario Internacional Europa-Latinoamérica de Investigación y Formación en \\ Ciencias Ambientales (EULA), Universidad de Concepción, Casilla 160-C, Concepción, Chile \\ ${ }^{2}$ Centro de Investigación en Ecosistemas de la Patagonia (CIEP), Coihaique, Chile \\ ${ }^{3}$ Departamento de Administración de Recursos Hídricos, Unidad de Obras Mayores, Dirección General de Aguas, \\ Santiago, Chile \\ ${ }^{4}$ Centro de Estudios Ambientales CIEMA, Universidad Nacional de Ingeniería, Managua, Nicaragua \\ * Autor correspondiente: cmoyap@udec.cl
}

\begin{abstract}
RESUMEN
En enero de 2006 se estudiaron los patrones espaciales de distribución de comunidades de macroinvertebrados bentónicos de la cuenca hidrográfica del río Baker $\left(45^{\circ} 50^{\prime} \mathrm{O}\right.$ y $\left.47^{\circ} 55^{\prime} \mathrm{S}\right)$ y los principales factores controladores, intentando cubrir la mayor variedad de ecosistemas lóticos. Para llevar a cabo el estudio se seleccionaron 27 estaciones de muestreo ubicadas en las diferentes subcuencas del río. En cada estación se realizó una caracterización fisicoquímica del agua (conductividad, oxígeno disuelto, pH, temperatura y turbidez), y se documentaron las características del tramo de río (e.g. ancho del cauce y tipo de sedimento) e información cartográfica utilizando un sistema de información geográfica (SIG). Se identificaron un total de 51 taxa que correspondieron en su mayoría a larvas de insectos $(80 \%)$. Los grupos con mayor riqueza fueron los órdenes Ephemeroptera (15 taxa), Plecoptera (8 taxa) y Trichoptera (8 taxa). Los análisis de clasificación y ordenación realizados con los datos de abundancia, permitieron reconocer siete grupos de estaciones diferentes $(\mathrm{A}-\mathrm{F})$ que fueron estadísticamente significativos $(\mathrm{P}<0.05)$. El análisis de regresión múltiple, muestra que la variable ambiental que mejor explica los patrones biológicos observados, fue el porcentaje de cobertura de bolones, explicando el $44 \%$ de la varianza de los datos biológicos $\left(\mathrm{r}^{2}=0.44 \mathrm{P}<0.05\right)$.
\end{abstract}

Palabras clave: cuenca del río Baker, hábitat fluvial, macroinvertebrados bentónicos, número de Froude, ríos patagónicos.

\begin{abstract}
In January of 2006 we studied the distributional patterns of benthic macroinvertebrate communities of the Baker river basin $\left(45^{\circ} 50^{\prime} \mathrm{O}\right.$ and $\left.47^{\circ} 55^{\prime} \mathrm{S}\right)$ and their main controlling factors trying to cover the greater variety of the lotic ecosystems. To carry out the study, 27 sampling stations were located in the different sub basins of the river. In each station, physical-chemical parameters of the column of water were quantified (conductivity, dissolved oxygen, $\mathrm{pH}$, temperature and turbidity), and documented characteristics of the segment (e.g. wide of the channel and sediment type) and cartographic information using a geographic information system (GIS) and complemented with cartographic information using GIS. Identified a total of 51 taxa, are mostly insect larvae $(80 \%)$. The groups most richness were orders Ephemeroptera (15 taxa), Plecoptera (8 taxa) and Trichoptera (8 taxa). The classification and ordination analyses carried out with the data of abundance, allowed to recognize seven groups stations $(A-F)$ statistically significant $(\mathrm{P}<0.05)$. The multiple regression analysis, showed that the environmental variable that accounts for the observed biological patterns, was coverage cobble, explaining $45.1 \%$ the variance of the biological data $\left(r^{2}=0.44 \mathrm{P}<0.05\right)$.
\end{abstract}

Key words: Baker river basin, benthic macroinvertebrates, fluvial habitat, Froude number, Patagonian rivers. 


\section{INTRODUCCIÓN}

Uno de los objetivos fundamentales de la ecología de ríos es entender los patrones de biodiversidad de los organismos y cuáles son los principales factores ambientales que influencian la distribución de las especies. Para entender dichos patrones es fundamental el estudio de áreas del planeta, que estén escasamente intervenidas (Oyanedel et al. 2008). La Patagonia chilena incluye los sistemas hídricos más complejos de Sudamérica y los menos intervenidos del planeta (Martínez-Harms \& Gajardo 2008, Carrasco et al. 2002). Dentro de este territorio, se localiza la cuenca del Río Baker ( $45^{\circ} 50^{\prime} \mathrm{O}$ y $47^{\circ} 55^{\prime} \mathrm{S}$ ), la cual posee un frágil y valioso patrimonio ambiental constituido por una gran variedad de ecosistemas lóticos, lénticos y estuarinos (Cruces et al. 1999). Ubicada al oriente de la cordillera andina Patagónica, abarca una extensión de 26,726 km² de los cuales $5,850 \mathrm{~km}^{2}$ se ubican en territorio argentino, siendo la segunda cuenca en cuanto a extensión después del Loa y el río de mayor caudal dentro de Chile (IGM 1984). Dentro de esta cuenca se emplaza el lago más grande del país, y el segundo de Sudamérica, después del Titicaca, lago General Carrera (denominado lago Buenos Aires en territorio argentino). Este lago es de origen glacial y posee una superficie total de $1,848 \mathrm{~km}^{2}$ teniendo este un importante efecto regulador respecto a las tasas de descarga del río Baker. En este territorio existen diferentes subcuencas hidrográficas, y considerando la variabilidad en el régimen de caudal, se observa una marcada diferencia entre los ríos que drenan los aportes nivo-glaciares de Campo de Hielo Norte, a diferencia de los ríos de la vertiente oriental, sujetos a climas transandinos y esteparios fríos (DGA 2005). La cuenca del río Baker ocupa el ecotono entre la zona climática templada septentrional y el dominio austral y presenta un marcado acento antártico (Fuenzalida 1965).

A esta rigurosidad climática, la cual es creciente de oeste a este y de norte a sur, se agregan las características de un relieve montañoso abrupto y fragmentado por las glaciaciones pleistocénicas (Naruse \& Aniya 1995, Carrasco et al. 2002). Durante la era glaciar, se iniciaba aquí parte del dominio de los grandes casquetes glaciares continentales de América del Sur, elemento determinante en su actual configuración geográfica (Naruse \& Aniya 1995). Aun hoy en día, dados los patrones de circulación atmosférica y oceánica del Pacífico Sur, la región conserva extensos campos de hielo, situación única en el mundo a esta latitud (Carrasco et al. 2002, Rosenblüth et al. 1998).

Los ciclos glaciales no solamente han modelado las características físicas de las cuencas, sino que produjeron variaciones profundas en la estructura de las comunidades biológicas presentes en las distintas unidades geográficas del mundo (Hewitt 2000), debido a la alternancia de fragmentación y coalescencia de las áreas sometidas a la actividad glacial (Connor 1986). Como resultado de la dinámica glacial pasada y actual, existe una enorme diversidad de ecosistemas y organismos (Hewitt 1996, Bond et al. 1993, Heinrich 1988), especialmente aquellos taxa de las regiones montañosas templadas (e.g. Taberlet \& Cheddadi 2002), como es el caso de la ecorregión patagónica, donde se ha constatado un número importante de especies endémicas de flora terrestre y fauna de vertebrados.

La biodiversidad dulceacuícola patagónica es una de las menos estudiadas del país y todavía quedan enormes vacíos de conocimiento, lo cual ha quedado en evidencia luego de recientes compilaciones sobre diferentes taxa, e.g. microalgas (Parra 2006, Rivera 2006), macrófitas (Hauenstein 2006), protozoos planctónicos (Woelfl 2006), tecamebianos (Zapata 2006), zooplancton (Villalobos 2006), crustáceos malacostracos (Jara et al. 2006), efemerópteros (Camousseight 2006), plecópteros (Vera \& Camousseight 2006), tricópteros (Rojas 2006), coleópteros (Jerez \& Moroni 2006), bivalvos (Parada \& Peredo 2006), gasterópodos (Valdovinos 2006), briozoos (Orellana 2006), peces (Habit et al. 2006), anfibios (Ortiz 2006), aves (Victoriano et al. 2006) y parásitos de organismos dulceacuícolas (Olmos \& Muñoz 2006). Sin embargo, con los antecedentes existentes se ha podido constatar un elevado grado de endemismo y patrones biogeográficos particulares, en el caso de los macroinvertebrados bentónicos fluviales (Oyanedel et al. 2008, Valdovinos 2006).

Los macroinvertebrados patagónicos han sido estudiados principalmente en ríos argentinos, destacándose los aportes de Pessacq \& Miserendino (2008), Miserendino (2006), Miserendino \& Pizzolon (2000) y Albariño 
(1997), los cuales analizaron la diversidad y los patrones de distribución de Plecoptera y/o Ephemerotera. Miserendino \& Pizzolon (2004), Miserendino (2001), Velásquez \& Miserendino (2003ab), Miserendino (1995) y Lopretto \& Tell (1995) quienes abordaron los aspectos funcionales y ecológicos de los sistemas lóticos patagónicos. La composición, abundancia y el rol de la temperatura ambiental sobre los patrones de emergencia, fueron estudiados en ensambles de quironómidos por García \& Añón-Suárez (2007). Finalmente, hay que destacar los hallazgos de nuevas especies de Plecoptera de McLellan et al. 2006.

En el noroeste de la Patagonia los antecedentes existentes son escasos, existiendo solamente datos acerca de taxa de macroinvertebrados bentónicos que forman parte de la dieta de peces (Vila et al. 1999, Milano et al. 2002, de Villalobos et al. 2008), y de la diversidad del orden Plecoptera existente en la cuenca del río Aisén, principalmente (Campos et al. 1984).

La estructura de las comunidades de invertebrados fluviales, en esta misma cuenca, fue estudiada por Oyanedel et al. (2008), quienes detectaron que ciertas variables hidráulicas son factores estructuradores importantes dentro de estas comunidades. En cambio, no existen estudios taxonómicos y ecológicos sobre los invertebrados fluviales del suroeste de la Patagonia, tal como ocurre en la cuenca del río Baker. Dado este marco, el presente estudio, propone analizar la distribución espacial de los macroinvertebrados bentónicos de la cuenca del río Baker, identificando los principales factores ambientales que determinan la configuración espacial de estas comunidades.

\section{MÉTODOS}

\section{Área de estudio}

Las muestras se obtuvieron durante el verano de 2006 en la cuenca hidrográfica del río Baker $\left(45^{\circ} 50^{\prime}\right.$ O y $\left.47^{\circ} 55^{\prime}, \mathrm{S}\right)$. Se muestrearon 27 estaciones que se distribuyeron en las diferentes subcuencas intentado cubrir la mayor diversidad de ecosistemas lóticos, abarcando ríos con diferentes áreas de drenaje, distintos microclimas y tipos de cubierta vegetal (Fig. 1).

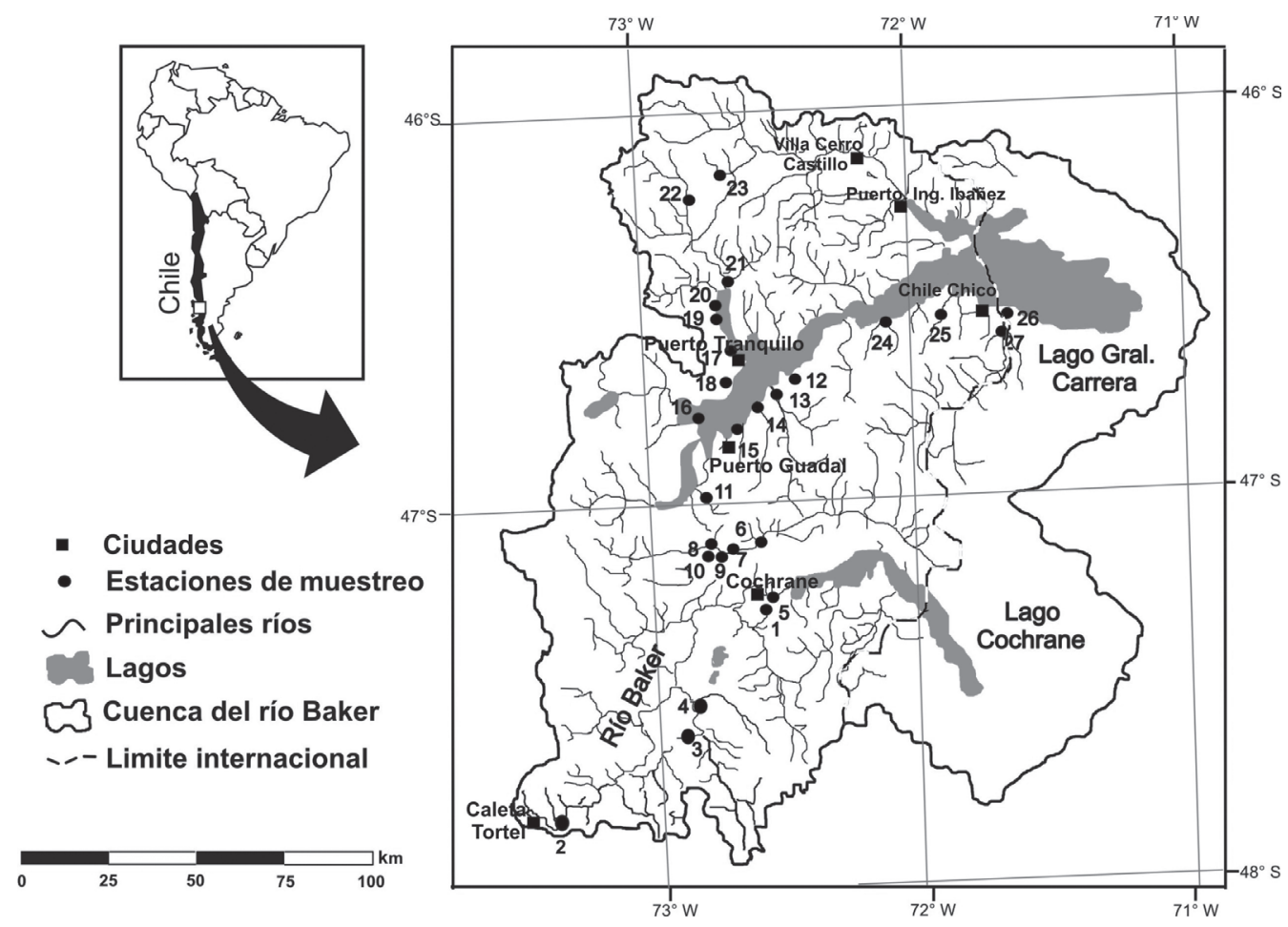

Fig. 1: Localización de las 27 estaciones de muestreo de la cuenca del río Baker. 
De acuerdo a Fuenzalida (1965), en la cuenca se pueden diferenciar cuatro tipos climáticos principales: a) Clima templado, frío lluvioso; b) Clima andino con degeneración estepárica; c) Clima de estepa frío y d) Clima de hielo de altura. En relación al relieve, Niemeyer et al. (1984) reconocen tres unidades geológicas, desde este hacia el oeste, estas son: a) Cordillera principal; b) Zona extraandina, parcialmente representada en Chile, pero con amplio desarrollo en Argentina y c) Precordillera, con alturas que no sobrepasan los $2.000 \mathrm{~m}$. y en ella se emplazan grandes lagos de origen glacial (e.g. lago General Carrera).

La vegetación del área está compuesta por Nothofagus antartica G. Forster (ñire), es la especie arbórea de distribución más amplia en la cuenca, formando bosques junto a Nothofagus dombeyi Mirb. (coihue) y Nothofagus pumilio Poepp et Endl. (lenga), hacia el norte, mientras que hacia el sur de la cuenca forma bosques con Pilgerodendron uviferum Don. (ciprés de las Guaitecas), encontrándose además, bosques de Nothofagus nitida Phil. (coihue de Chiloé) a una menor altitud. La vegetación esteparia, se encuentra dominada por Mulinun spinosus Cav. (palo negro), Festuca magellanica Lam. (coirón) y Empetrum rubrum Vahl ex Willd (brecillo). El matorral está compuesto principalmente por Colletia hystrix Clos. (yaqui), N. antartica G. Forster (ñire) que se presenta como arbusto, Embothrium coccineum J.R. et G. Forster (notro) y Gaultheria mucronata (L.f.) Hook. et Arn (chaura) (Rodríguez et al. 2008).

\section{Caracterización del hábitat fluvial}

En cada sitio de muestreo se registró in situ la temperatura del agua $\left({ }^{\circ} \mathrm{C}\right)$, la conductividad $(\mu$ $\left.\mathrm{S} \mathrm{cm}^{-1}\right)$, el $\mathrm{pH}$ y el oxígeno disuelto $\left(\mathrm{mg} \mathrm{L}^{-1}\right)$, los cuales fueron medidos con un sonda multiparamétrica HI 9828. La turbidez (NTU) fue medida con un turbidímetro HI 93703 C. La velocidad media de la corriente $\left(\mathrm{m} \mathrm{s}^{-1}\right)$, se determinó con un flujómetro Gurley, el ancho del río, se midió con una huincha y la profundidad con una vara graduada $(\mathrm{cm})$. La pendiente se estimó visualmente y se clasificó en cuatro categorías (muy alta $=4$, alta $=3$, moderada $=2$ y baja $=1)$ siendo superiores a $30^{\circ}$ las pendientes muy altas, altas entre $30^{\circ}$ $20^{\circ}$, moderadas entre $20^{\circ}-10^{\circ}$ y menores a $10^{\circ}$ las pendientes bajas.
Para este estudio, se utilizó cartografía 1:50,000 (IGM) y digitalizada en SIG. El área de drenaje $\left(\mathrm{km}^{2}\right)$ y la elevación media $(\mathrm{m})$ de cada subcuenca, fue calculada a través de un Modelo de Elevación Digital (MED), con pixel de aproximadamente $77 \mathrm{~m}$. La cobertura vegetal se determinó a través del Catastro de bosque nativo realizado por la Corporación Nacional Forestal (CONAF 1999). El procesamiento y análisis de los datos fue realizado utilizando el software ArcGIS 9.2.

Siguiendo a Statzner et al. (1988), la velocidad de la corriente y la profundidad media de cada punto de muestreo fueron combinadas para calcular el promedio del número de Froude (FROU), que corresponde a la razón entre fuerzas inerciales y gravitacionales. Este es un muy buen indicador del estrés hidráulico para los macroinvertebrados bentónicos: FROU $=\mathrm{U} /$ $\left(\begin{array}{ll}g & D\end{array}\right)^{0.5}$, donde $U=$ velocidad media de la corriente; $g$ = fuerza gravitacional $\left(9.8 \mathrm{~m} \mathrm{~s}^{-2}\right) \mathrm{y}$ $\mathrm{D}=$ profundidad media del agua .

Se realizó una caracterización visual de la composición del substrato, calculada utilizando un cuadrante metálico de $1 \mathrm{~m}^{2}$. Categorizándolos en: a) bolones $(>20 \mathrm{~cm})$, b) gravas $(0.2-20 \mathrm{~cm})$ y c) arenas y fangos $(<0.2 \mathrm{~cm}$ ) (Folk 1980). Los resultados se expresaron como la proporción de las distintas fracciones en un área de determinada $\left(1 \mathrm{~m}^{2}\right)$. Con estos datos se calculó un índice de diversidad de sustrato (SUDI), de acuerdo a: SUDI $=1 /\left(\sum \mathrm{p}_{\mathrm{i}}^{2}\right)$, donde $\mathrm{p}_{\mathrm{i}}$ es la proporción de la i-ésima categoría de sustrato (Castella et al. 2001). Los bolones fueron medidos empleando una regla milimetrada para determinar el diámetro máximo y promedio (Hulings \& Gray 1971).

\section{Macroinvertebrados bentónicos}

Las muestras de macroinvertebrados bentónicos, se obtuvieron con una red de mano de $20 \mathrm{~cm}$ de diámetro y $500 \mu \mathrm{m}$ de abertura de malla. En cada sitio se obtuvieron seis muestras con similares esfuerzos, abarcando un área aproximada de $0.6 \mathrm{~m}^{2}$ para cada una de las muestras. Las muestras fueron conservadas en etanol al $75 \%$, y luego trasladadas a laboratorio para su posterior separación e identificación de los organismos hasta el nivel taxonómico más bajo posible, empleando para ello un estereomicroscopio Zeiss Stemi SR. Los diferentes taxa se identificaron siguiendo a: Wais (1987), Lugo-Ortiz \& McCafferty (1998), 
Fernández \& Domínguez (2001), Romero (2001), González (2003), Heckman (2003), McLellan et al. (2005), Domínguez et al. (2006) y Sganga \& Fontanarrosa (2006).

\section{Análisis de datos}

Las estaciones fueron comparadas considerando los datos físicos y químicos, empleando análisis multivariados de ordenamiento y clasificación. Se utilizó el programa PRIMER versión 6.1.2 - Plymouth Routines in Multivariate Ecological Research (Clarke et al. 2005).

Los datos ambientales fueron primariamente transformados (raíz cuadrada) para luego construir una matriz de distancia Euclidiana, con la cual se construyó un dendrograma, empleando ligamiento completo como método de agrupamiento. Se realizó la prueba de Simproff sobre el dendrograma, para identificar los grupos estadísticamente significativos $(\mathrm{P}<0.05)$. Posteriormente, la matriz de distancia Euclidiana fue analizada utilizando escalamiento no métrico multidimensional (NMDS) como método de ordenamiento. Los datos biológicos fueron comparados considerando los datos de densidad promedio de macroinvertebrados bentónicos por estación. Estos datos fueron primariamente transformados $(\log \mathrm{x}+1)$ para construir la matriz de similitud de Bray-Curtis, cuyo dendrograma se graficó empleando ligamiento completo como método de agrupamiento. Se realizó la prueba de Simproff sobre el dendrograma para identificar los grupos estadísticamente significativos $(\mathrm{P}<$ 0.05). La matriz de similitud fue analizada utilizando NMDS como método de ordenación, representando en dos dimensiones la similitud de las estaciones de muestreo basadas en la abundancia y composición de las comunidades de macroinvertebrados bentónicos. Posteriormente, los valores de las dos dimensiones del NMDS y los parámetros ambientales, fueron analizados a través de regresión múltiple modelo estándar, tratando cada parámetro abiótico como una variable dependiente, y las coordenadas de la ordenación en cada eje del NMDS, los cuales son un resumen del ordenamiento de los datos biológicos, como las variables independientes (Field et al. 1982). Los parámetros ambientales analizados se detallan en la Tabla 1.

Con el objeto de comparar las estaciones de muestreo en base a sus atributos comunitarios, se analizaron: riqueza específica, abundancia total, el índice de diversidad de Shannon y Equidad (J'), empleando el software PRIMER versión 6.1.2.

\section{RESULTADOS}

\section{Caracterización del hábitat fluvial}

En la Tabla 1 se presenta la caracterización de las 27 estaciones de muestreo en la cuenca del río Baker (cobertura vegetal, parámetros morfométricos, hidrodinámicos, sedimentológicos y fisicoquímicos de calidad de agua). Los análisis de clasificación y ordenación realizados con los datos ambientales, permiten reconocer siete grupos significativamente distintos $(\mathrm{P}<0.05)$ de estaciones de muestreo (A-G) a una distancia euclidiana de 8.1 (Figs. 2A y 2B). Para el grupo A (estaciones ubicadas entre Puerto Guadal y Villa Cerro Castillo, a excepción de una estación 9 ubicada en la parte media de la cuenca, entre Cochrane y Puerto Bertrand), se observaron valores intermedios de cada parámetro analizado. El grupo B (estaciones 23 y 27, Chile Chico y Villa Cerro Castillo, respectivamente) se diferenció marcadamente de los restantes grupos, por su relativamente elevada diversidad de sustrato (SUDI $=2.63)$. El grupo C (estaciones ubicadas en la parte media y media-alta de la cuenca, entre Cochrane y Puerto Guadal), se caracterizó porque en la mayoría sus estaciones agrupadas, se observó una mayor cobertura de grava $(80-60 \%)$. D agrupa las estaciones localizadas en la parte alta de la cuenca (Chile Chico), cuyas subcuencas presentaron una mayor elevación media (419.5 $318 \mathrm{~m}$ ). E corresponde a un solo río, ubicado en la parte media-baja de la cuenca (salida de Cochrane, camino a Caleta Tortel), que se caracterizó por su elevada conductividad $\left(238 \mu \mathrm{S}^{-1}\right)$. Las estaciones del grupo $\mathrm{F}$, se encuentran en la parte baja de la cuenca, próximas a Caleta Tortel, correspondiendo a tres subcuencas distintas, con bosque nativo dominado por ciprés de las Guaitecas y ñire, principalmente. Por último, las estaciones agrupadas en $\mathrm{G}$, se ubicaron en la parte media-baja de la cuenca del Baker, y tuvieron una mayor área de drenaje $\left(5,096.1-577.6 \mathrm{~km}^{2}\right)$ que las demás estaciones, lo que se relacionó directamente con el mayor ancho promedio del cauce $(130-45 \mathrm{~m})$ y la mayor profundidad media (1.5 - 0.6 m). (Figs. 1, 2A y 2B) 


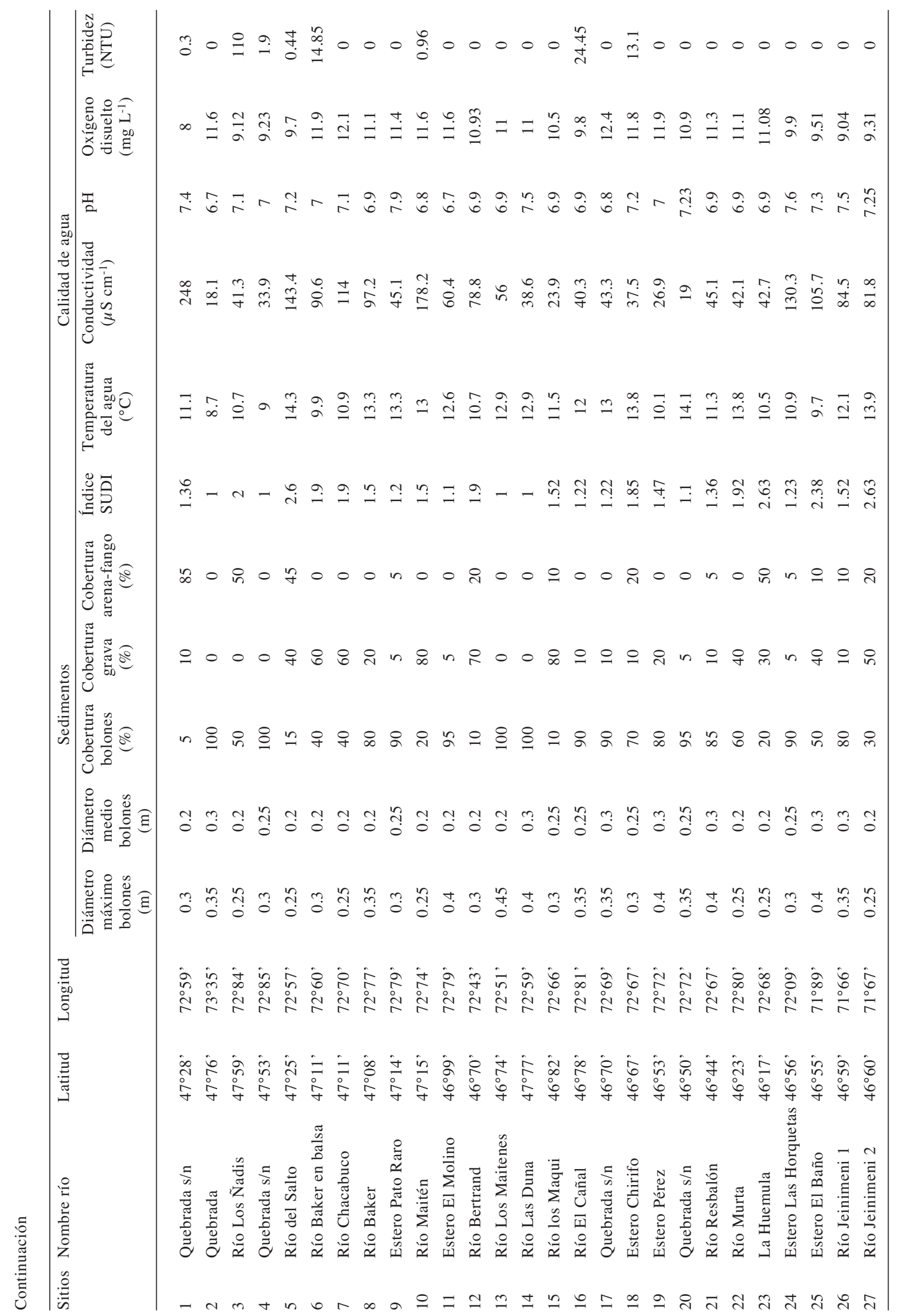




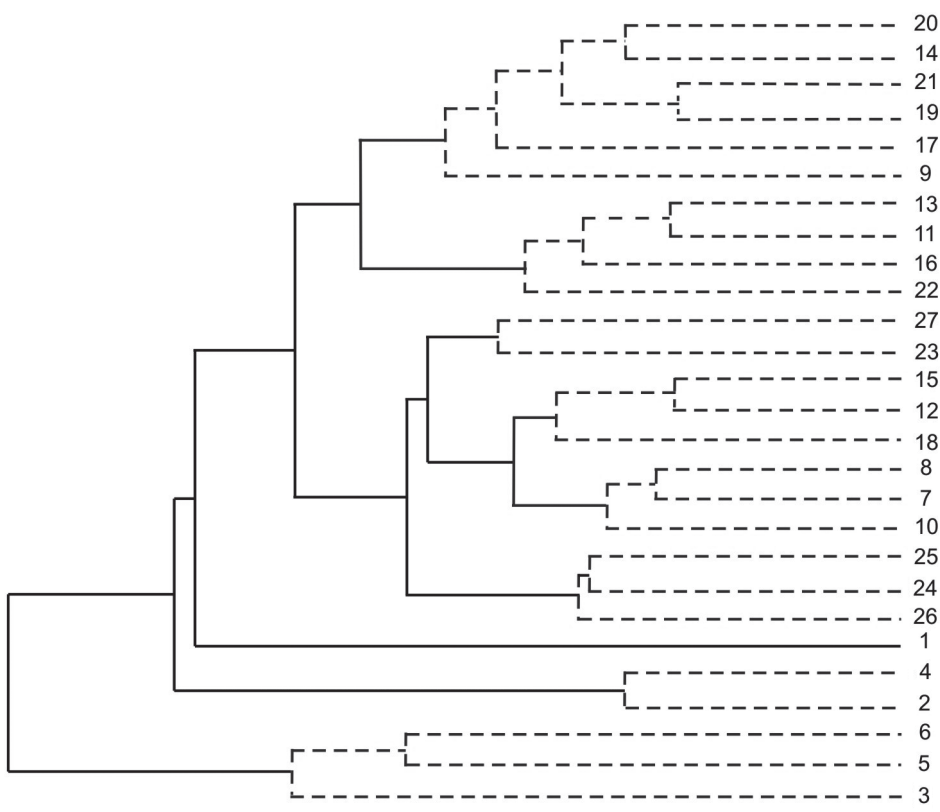

Grupo A

Grupo B

Grupo C

Grupo D

| Grupo E

Grupo F

Grupo G

15

(A)

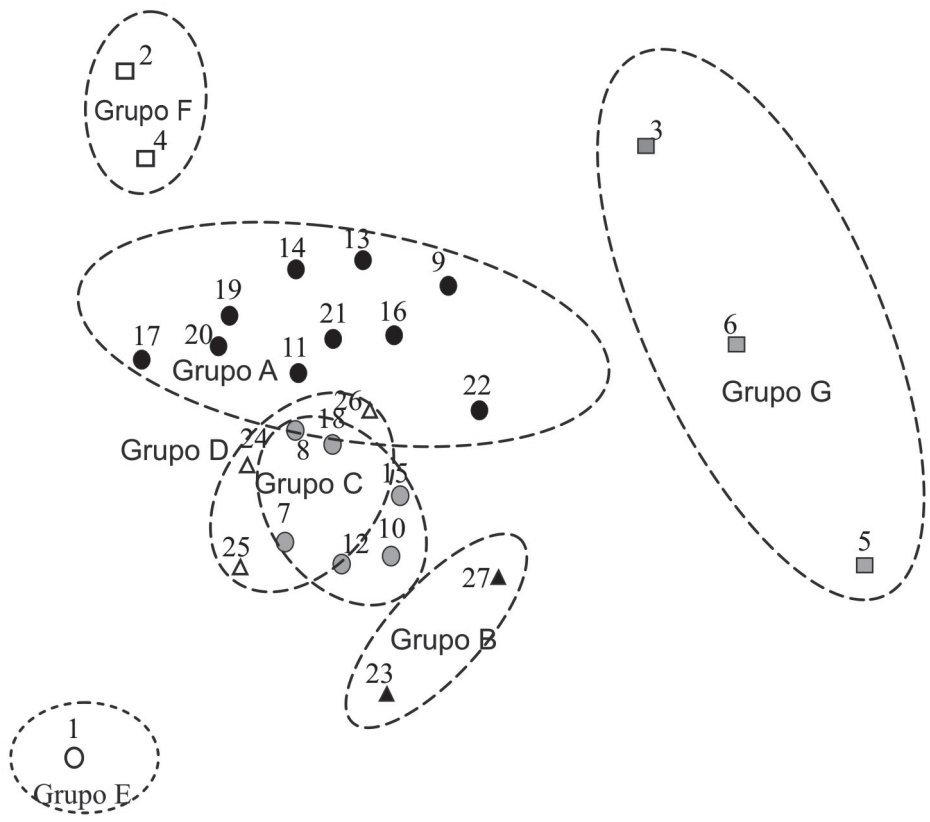

Stress: 0.15

(B)

Fig. 2: Clasificación y ordenamiento de las 27 estaciones de muestreo, mediante análisis de dendrograma (A) y escalamiento no métrico multidimensional (NMDS) (B), obtenidos sobre la base de la caracterización del hábitat fluvial (Tabla 1). Los grupos fueron definidos empleando el test Simprof $(\mathrm{P}<0.05)$. Los ejes no tienen leyendas por tener una escala relativa.

Classification and ordination of the 27 sampling sites using cluster analysis (A) and non-metric multidimensional scaling (NMDS) (B), based on fluvial habitat database (Table 1). The groups were defined using the Simprof test $(\mathrm{P}<0.05)$. Have no legends axes because is a relative scale. 


\section{Macroinvertebrados bentónicos}

Se identificaron un total de 51 taxa, la mayor parte de los cuales correspondieron a estados inmaduros de insectos $(80 \%)$. Dentro de estos, los de mayor riqueza específica fueron los órdenes Ephemeroptera (15 taxa), Plecoptera (8 taxa) y Trichoptera (8 taxa) y Diptera (7 taxa) (Tabla 2). Los análisis de clasificación y ordenación de las estaciones se realizaron con los datos de composición y abundancia a nivel taxonómico de especie y morfoespecie, permitiendo reconocer siete grupos estadísticamente diferentes $(\mathrm{P}<0.05)$ de estaciones de muestreo (A-G) (Figs. 3A y 3B).

Los datos exhibidos en la Tabla 3 muestran que las estaciones con mayor riqueza taxonómica, fueron la 12 y la 27 con 31 y 30 taxa respectivamente, las cuales están incluidas en el grupo A (Fig. 4A). Las estaciones de esta agrupación pertenecen todas a distintas subcuencas, excepto las estaciones 26 y 27 , ambas ubicadas en la subcuenca del río Jeinimeni. Además, la mayoría de las estaciones de este grupo se ubican en la parte alta y media de la cuenca a excepción de la estación 4 (río el Salto), ubicada en la parte media-baja. Al contrario, la estación 3 (perteneciente al grupo C) presentó menor riqueza (6 taxa). La mayoría de las estaciones de este grupo, se ubican en la parte media-alta de la cuenca del Baker (estaciones 9, 6, 17 y 3), solo la estación 3 (río Nadis) se encuentra en la parte baja de la cuenca.

En términos de abundancia, la estación 5 (grupo B) mostró el valor más elevado (511 ind. $\left.\mathrm{m}^{-2}\right)$. B reúne 2 estaciones de muestreo próximas entre sí, en la en la parte media-baja de la cuenca (estaciones 5 y 1). En cambio, el menor valor de abundancia (39 ind $\mathrm{m}^{-2}$ ), fue encontrado en las estaciones 2 (grupo E) y 20 (grupo G). Por otro lado, la riqueza taxonómica promediada por cada grupo de estaciones de muestreo, fue mayor en A y B, grupos que exhibieron un promedio de $18.14 \pm$ 8.6 y $18 \pm 4.3$ (promedio \pm desviación estándar) taxa respectivamente (Tabla 3). Contrariamente, el grupo con menor riqueza taxonómica fue E, con 8 taxa. Las estaciones agrupadas en E, se ubican en la parte baja de la cuenca cerca de Caleta Tortel (estación 2) y en su parte alta, cerca de Puerto Tranquilo (estación 19).
En cuanto a la abundancia, promediada por cada uno de los grupos anteriores, los valores más altos se observaron en $\mathrm{B}$ con $469.5 \pm 9.5$ ind. $\mathrm{m}^{-2}$ (promedio \pm desviación estándar), debido al número de individuos de Chironomidae y Hyallela sp. Abundancias importantes también se detectaron en el grupo A, debido al elevado número de individuos de Chironomidae. Las menores abundancias promedio se observaron en el grupo $G$, con $32.25 \pm 15.91$ ind. $\mathrm{m}^{-2}$ (promedio \pm desviación estándar), donde fue dominante la familia Simuliidae (Fig. 4A) . Por último, los valores de diversidad de Shannon (H') y Equidad (J') no mostraron grandes diferencias entre los grupos y dentro de cada grupo.

\section{Relación entre los macroinvertebrados bentóni- cos y las características del hábitat}

El ordenamiento de las estaciones de muestreo de acuerdo a la composición y abundancia de sus especies (NMDS), estuvo determinado por el porcentaje de bolones que componen el sustrato de fondo del lecho, variable que explicó el $44 \%$ de la varianza de los datos biológicos $\left(\mathrm{r}^{2}=0.44\right.$ $\mathrm{P}<0.001)$, y además se correlacionó significativamente con la parámetros comunitarios de abundancia $\left(\mathrm{r}^{2}=0.48, \mathrm{P}<\right.$ $0.001)$ y riqueza taxonómica $\left(\mathrm{r}^{2}=0.22, \mathrm{P}<\right.$ $0.05)$. Por otro lado, un $33 \%$ de la varianza de los datos biológicos estuvo explicada por la conductividad del agua $\left(\mathrm{r}^{2}=0.33, \mathrm{P}<0.001\right)$, al igual que el área de cobertura de estepa en la cuenca $\left(r^{2}=0.33, P<0.001\right)$. Las variables ambientales restantes explicaron entre un $25 \mathrm{y}$ un $17 \%$ de la varianza de los datos biológicos: área de cobertura de bosque nativo y matorral $\left(\mathrm{r}^{2}\right.$ $=0.25, \mathrm{P}<0.05)$, área de drenaje especifica $\left(\mathrm{r}^{2}=\right.$ $0.23, \mathrm{P}<0.05)$, porcentaje de grava $\left(\mathrm{r}^{2}=0.21, \mathrm{P}\right.$ $<0.05)$, velocidad de la corriente $\left(\mathrm{r}^{2}=0.21, \mathrm{P}<\right.$ $0.05)$, y el porcentaje de arena-fango $\left(\mathrm{r}^{2}=0.17\right.$, $\mathrm{P}<0.05$ ) (Tabla 4).

\section{DISCUSIÓN}

En el presente estudio, las técnicas de ordenación y clasificación utilizadas, permitieron identificar los factores que influyeron en la distribución espacial de las comunidades de macroinvertebrados bentónicos en la cuenca hidrográfica del Baker. 
TABLA 2

Listado taxonómico de macroinvertebrados bentónicos (> $500 \mu \mathrm{m}$, tamaño de los invertebrados) registrados en la Cuenca Hidrográfica del río Baker.

Taxonomic list of benthic macroinvertebrates (> $500 \mu \mathrm{m}$, size of invertebrates) recorded in the Baker River Basin

\begin{tabular}{|c|c|c|c|}
\hline Phylum (o Clase) & Clase (u Orden) & Familia (o grupo) & Especie (o grupo) \\
\hline Platyhelminthes & Turbellaria & Dugesiidae & Girardia sp. \\
\hline Annelida & Oligochaeta & Lumbriculidae & $\begin{array}{l}\text { Lumbriculidae sp. } \\
\text { Nais sp. }\end{array}$ \\
\hline \multirow[t]{3}{*}{ Mollusca } & Gastropoda & Amnicolidae & Littoridina sp. \\
\hline & & Chilinidae & Chilina sp. \\
\hline & & Lymnaeidae & Lymnaea viator (dOrbigny, 1835) \\
\hline \multirow[t]{3}{*}{ Chelicerata } & Acari & & Acari sp. 1 \\
\hline & & & Acari sp. 2 \\
\hline & & & Acari sp. 3 \\
\hline Crustacea & Amphipoda & Hyallelidae & Hyallela curvispina (Shoemaker, 1942) \\
\hline \multirow[t]{41}{*}{ Insecta } & Odonata & & Odonata sp. \\
\hline & Ephemeroptera & Leptophlebiidae & Leptophlebiidae sp. \\
\hline & & & Meridialaris diguillina (Navás, 1918) \\
\hline & & & Meridialaris spina (Pescador \& Peters, 1986) \\
\hline & & & Meridialaris laminata (Ulmer, 1920) \\
\hline & & & Meridialaris chiloeensis (Demoulin, 1955) \\
\hline & & & Meridialaris sp. \\
\hline & & & Penaphlebia fulvipes (Needham \& Murphy, 1924) \\
\hline & & & Penaphlebia sp. \\
\hline & & & Massartellopsis irarrazavali (Demoulin,1955) \\
\hline & & & Massartellopsis sp. \\
\hline & & Baetidae & Baetidae sp. \\
\hline & & & Andesiops sp. \\
\hline & & & Andesiops torrens (Lugo-Ortiz \& McCafferty, 1999) \\
\hline & & & Andesiops ardua (Lugo-Ortiz \& McCafferty, 1999) \\
\hline & & & Andesiops peruvianus (Ulmer, 1920) \\
\hline & Plecoptera & Gripopterygidae & Gripopterygidae spp. \\
\hline & & & Antarctoperlinae sp. 1 \\
\hline & & & Limnoperla jaffueli (Navás, 1928) \\
\hline & & & Senzilloides panguipullii (Navás, 1928) \\
\hline & & & Notoperla sp. \\
\hline & & & Neuroperla schedingi (Navás, 1929) \\
\hline & & Notonemouridae & Udamoceria sp. \\
\hline & & Austroperlidae & Klapopteryx kuscheli (Illies, 1960) \\
\hline & Trichoptera & Limnephilidae & Limnephilidae sp. \\
\hline & & Hydropsychidae & Smicridea annulicornis (Blanchard) \\
\hline & & Hydroptilidae & Neotrichia sp. \\
\hline & & Policentropodidae & Policetropodidae sp. \\
\hline & & Leptoceridae & Brachysetodes sp. \\
\hline & & & Leptoceridae sp. \\
\hline & & Philopotamidae & Philopotamidae sp. \\
\hline & & Hydrobiosidae & Hydrobiosidae sp. \\
\hline & Coleoptera & Elmidae & Elmidae spp. \\
\hline & & Helodidae & Helodidae sp. \\
\hline & Diptera & Chironomidae & Chironomidae spp. \\
\hline & & Empididae & Empididae sp. \\
\hline & & Athericidae & Athericidae sp. \\
\hline & & Simuliidae & Simuliidae sp. \\
\hline & & Ceratopogonidae & Ceratopogonidae sp. \\
\hline & & Tipulidae & Tipulidae sp. \\
\hline & & Blephariceridae & Blephariceridae sp. \\
\hline
\end{tabular}



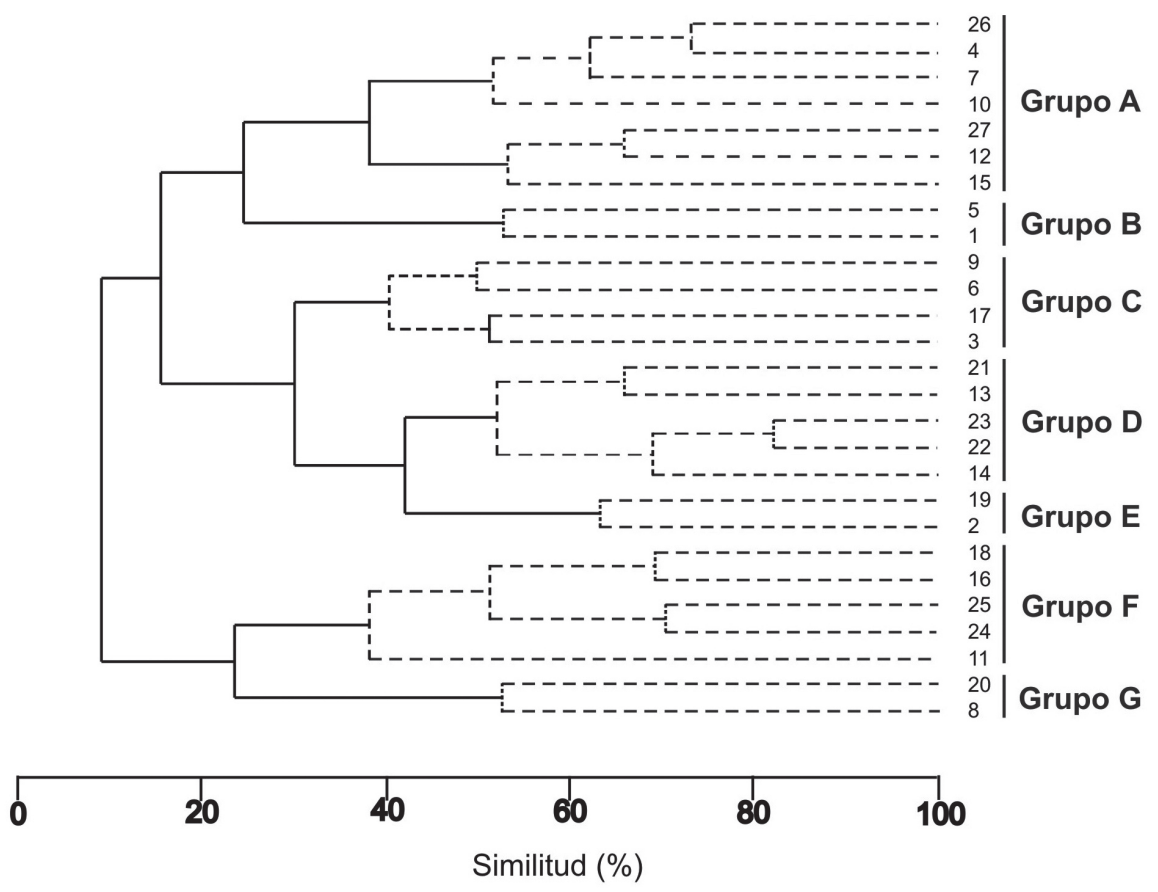

(A)

Stress: 0.19

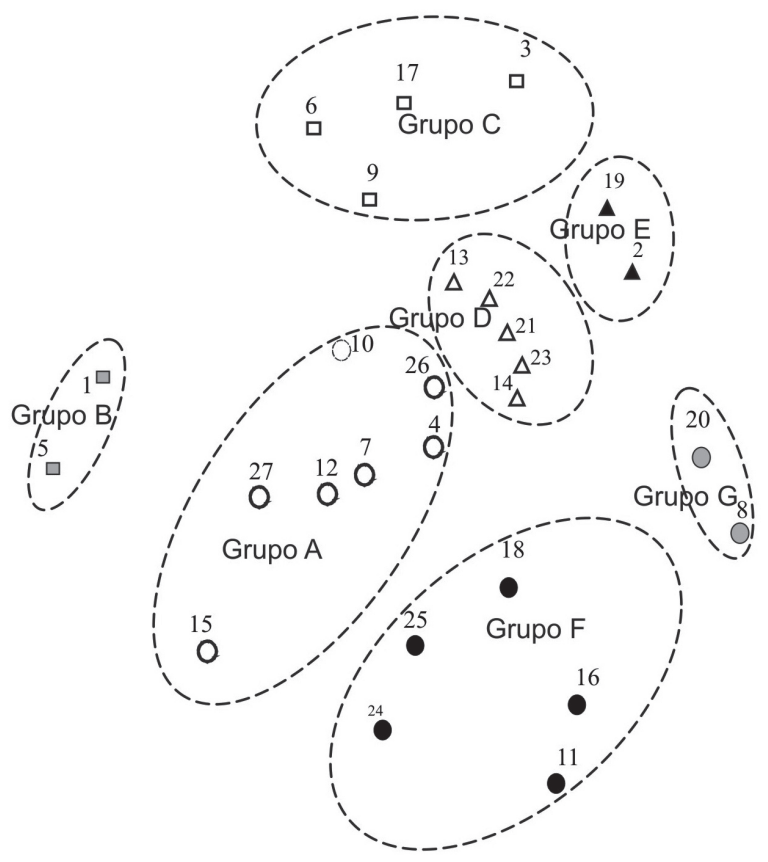

(B)

Fig. 3: Clasificación y ordenamiento de las 27 estaciones de muestreo mediante análisis de dendrograma (A) y escalamiento no métrico multidimensional (NMDS) (B). Ambos análisis se realizaron sobre la base de una matriz de disimilitud de Bray \& Curtis, calculada con los datos de abundancia (ind. $\left.\mathrm{m}^{-2}\right)$. Los grupos fueron definidos empleando el test Simprof $(\mathrm{P}<0.05)$.

Classification and ordination of the 27 sampling sites through cluster analysis (A) and non-metric multidimensional scaling (NMDS) (B). Both analyses were obtained on a Bray \& Curtis dissimilarity matrix of abundance data (ind. $\mathrm{m}^{-2}$ ). The groups were defined using Simprof test $(\mathrm{P}<0.05)$ 


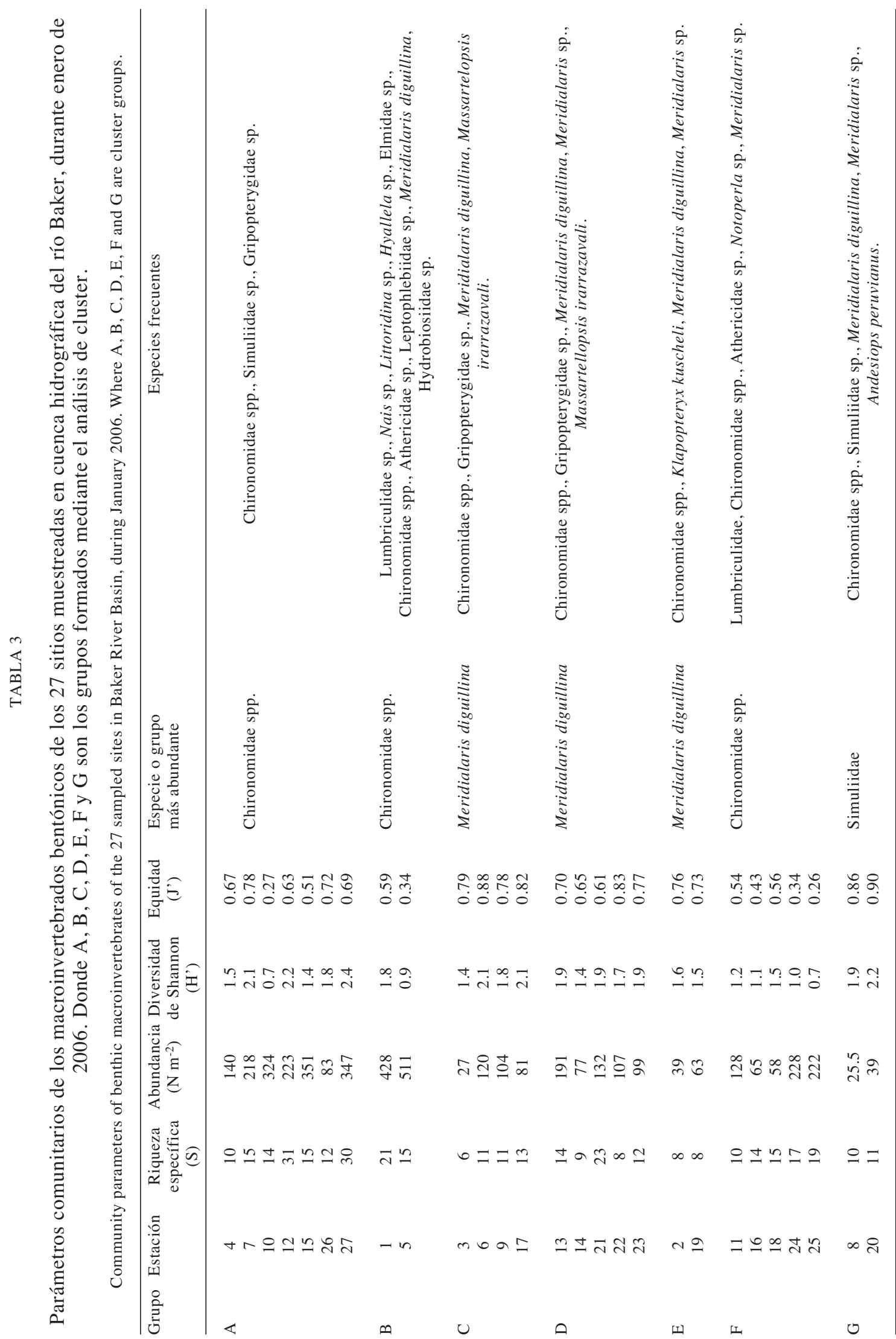




\section{Grupos}
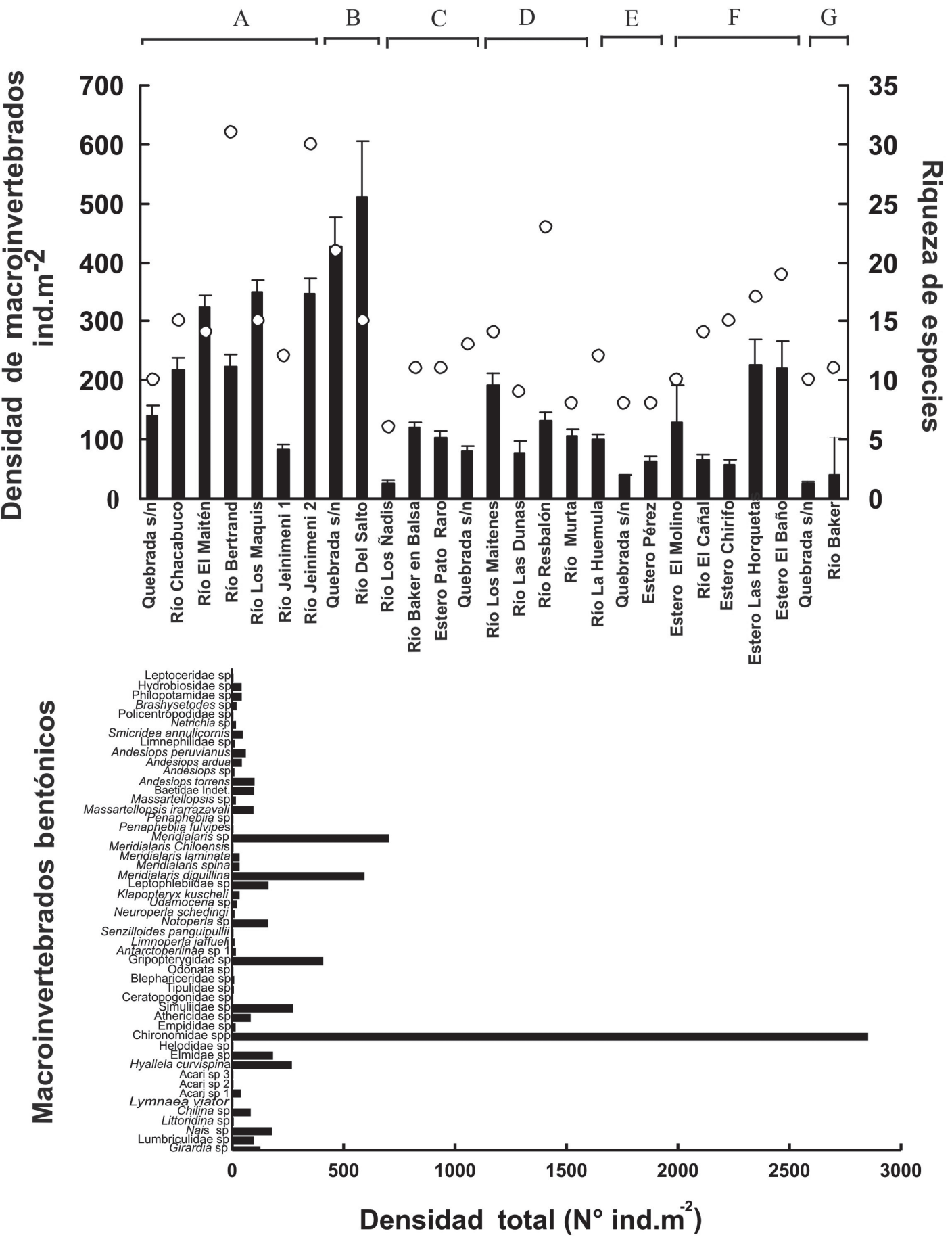

(B)

Fig. 4: (A) Riqueza de especies (círculos) y densidad de individuos promedio (barras) \pm DE, mostrando también los grupos (A-G), en los cuales se reúnen cada uno de los ríos muestreados. (B) Densidad total por taxón en los 27 ríos muestreados en la cuenca del Baker.

(A) Species richness (circles) and average individual density (bars) \pm SD, showing groups (A-G), which is aggregated each sampled rivers. (B) Total density by taxon in the 27 streams sampled in Baker river basin. 
TABLA 4

Regresión múltiple entre las variables del hábitat y los valores de los dos primeros ejes del NMDS (donde el primer eje es significativo) de la comparación de las estaciones de muestreo en base a los datos de abundancia. $* * \mathrm{P}<0.001=$ muy significativo; $* \mathrm{P}<0.05=$ significativo; $\mathrm{n} . \mathrm{s} .=$ no significativo.

\begin{tabular}{|c|c|c|c|c|}
\hline Variable & $\mathrm{r}^{2}$ & $\mathrm{~F}$ & g.1 & $\mathrm{p}$ \\
\hline (\%) bolones & $(-) 0.44$ & 11.3 & 2.24 & $* *$ \\
\hline Conductividad $\left(\mu \mathrm{S} \mathrm{cm}^{-1}\right)$ & (-) 0.33 & 7.36 & 2.24 & $* *$ \\
\hline Área cobertura estepa $\left(\mathrm{km}^{2}\right)$ & 0.27 & 6.00 & 2.24 & $* *$ \\
\hline Área cobertura matorral $\left(\mathrm{km}^{2}\right)$ & 0.25 & 5.40 & 2.24 & $*$ \\
\hline Área cobertura bosque nativo $\left(\mathrm{km}^{2}\right)$ & 0.25 & 5.35 & 2.24 & $*$ \\
\hline Área de drenaje específica $\left(\mathrm{km}^{2}\right)$ & 0.23 & 4.93 & 2.24 & $*$ \\
\hline (\%) grava & 0.21 & 4.48 & 2.24 & $*$ \\
\hline Velocidad media $\left(\mathrm{m} \mathrm{s}^{-1}\right)$ & 0.20 & 4.43 & 2.24 & $*$ \\
\hline$(\%)$ arena y fango & (-) 0.17 & 3.57 & 2.24 & $*$ \\
\hline Diámetro máximo de bolones (m) & 0.15 & 3.19 & 2.24 & n.s. \\
\hline Ancho medio del cauce (m) & 0.12 & 2.70 & 2.24 & n.s. \\
\hline Oxígeno disuelto $\left(\mathrm{mg} \mathrm{L}^{-1}\right)$ & 0.11 & 2.57 & 2.24 & n.s. \\
\hline Índice SUDI & 0.08 & 2.15 & 2.24 & n.s. \\
\hline Turbidez & 0.034 & 1.47 & 2.24 & n.s. \\
\hline Pendiente & 0.02 & 1.30 & 2.24 & n.s. \\
\hline Diámetro medio de bolones (m) & (-) 0.02 & 1.27 & 2.24 & n.s. \\
\hline Altura (m) & 0.015 & 1.20 & 2.24 & n.s. \\
\hline Profundidad media (m) & 0.013 & 1.18 & 2.24 & n.s. \\
\hline Número de Froude & 0.003 & 1.03 & 2.24 & n.s. \\
\hline $\mathrm{pH}$ & - & 0.90 & 2.24 & n.s. \\
\hline Temperatura $\left({ }^{\circ} \mathrm{C}\right)$ & - & 0.18 & 2.24 & n.s. \\
\hline
\end{tabular}

La caracterización física de los hábitats lóticos y de la calidad del agua en la cuenca de río Baker, permitió identificar estaciones de muestreo agrupadas de acuerdo a las diferencias físicas naturales existentes en la cuenca, las cuales varían enormemente al interior de la cuenca (Doisy \& Rabeni 2001, Jowett 2003). De este modo, se encontraron estaciones agrupadas debido a la mayor elevación media de su cuenca, como aquellas localizadas en Chile Chico, área que se caracteriza por tener un microclima, donde predomina una baja precipitación media y con dominio de vegetación de tipo estepa y matorral, dada por su cercanía a la vertiente oriental, zona limítrofe con Argentina (CONAF 1999). Hacia la parte baja de la cuenca, las estaciones de muestreo se caracterizaron por presentar bosques, desarrollados en una de las zonas más pluviosas dentro de la cuenca. Por otro lado, las mayores áreas de drenaje del grupo $\mathrm{G}$, están caracterizadas por la presencia del río Baker en su parte media y los ríos Nadis, y el Salto en la parte media-baja de la cuenca, siendo estos últimos dos importantes tributarios del Baker. Un río de bajo orden que conforma a E, presentó elevada conductividad, probablemente causada por el desarrollo de actividades agroganaderas en un sector muy cercano al poblado de Cochrane.

Las comunidades de macroinvertebrados observadas en el presente estudio son similares en cuanto a su composición taxonómica a las reportadas en otras cuencas hidrográficas de la Patagonia argentina (Miserendino 2001), siendo los órdenes Ephemeroptera, Plecoptera, Trichoptera y Diptera, los más diversos y con mayor abundancia (Wais 1987, Wais \& Boneto 
1988, Wais 1990, Miserendino \& Pizzolon 2004). En la cuenca del río Aisén $\left(44^{\circ} 45^{\prime}\right.$ $\left.46^{\circ} 15^{\prime} \mathrm{S} ; 71^{\circ} 00^{\prime}-73^{\circ} 00^{\prime} \mathrm{O}\right)$ Campos et al. (1984) determinaron 12 especies de plecópteros, coincidentes con las registradas por Oyanedel et al. (2008), quienes también observaron a este orden como uno de los dominantes en las comunidades de macroinvertebrados bentónicos fluviales, junto a los Diptera y Ephemeroptera. En cambio, en el presente estudio, fueron determinadas ocho especies de Plecoptera, y también constituye un orden dominante en este tipo de comunidades. Lo antedicho concuerda con la disminución de la riqueza especifica hacia altas latitudes propuesta por Brayard et al. (2005) y, concordando con los obtenidos por C Valdovinos et al. (datos no publicados), para el caso de Plecoptera.

La mayor riqueza taxonómica de las estaciones 12 y 27 podría ser explicada por el alto índice de diversidad de sustrato observado en estas estaciones (SUDI), comparadas con el resto de los puntos muestreados. Esto es consistente con el hecho que la riqueza y productividad de los macroinvertebrados fluviales depende en gran medida de la heterogeneidad del hábitat bentónico constituido por el sustrato de fondo (Velásquez \& Miserendino 2003a). La estación 3 ubicada en el río Ñadis, hacia el sur de la cuenca, presenta la menor riqueza taxonómica. Esta estación es difícil de muestrear y su régimen hidrológico se encuentra muy influenciado por la mayor frecuencia e intensidad de precipitaciones en comparación con el resto de la cuenca (Niemeyer et al. 1984), existiendo crecidas que convierten el sustrato en un hábitat inestable lo que dificulta el asentamiento de los organismos. Para el grupo A (el de mayor riqueza taxonómica), los principales taxa que incidieron en el agrupamiento de las estaciones de muestreo (ver Fig. 3), fueron: Chironomidae, Simuliidae, Gripopterygidae spp., Notoperla sp., Meridialaris diguillina (Navás, 1918), Meridialaris sp. y Andesiops peruvianus (Ulmer, 1920). La mayoría de estas especies, y en particular Notoperla sp., pueden estar asociadas a ríos de cabecera, caracterizados por estar a mayor altura, por tener aguas muy oxigenadas y sometidos a periodos de congelamiento. Las estaciones de muestreo agrupadas en $\mathrm{C}$ (grupo con menor riqueza taxonómica), se caracterizan por presentar mayor abundancia de taxa como: Girardia sp., Chironomidae, Gripoterygidae spp., Meridialaris diguillina, siendo esta última una especie ramoneadora de algas perifíticas, presentes de manera abundante en las estaciones que forman parte del grupo C (Miserendino 1995).

Considerando una meso-escala espacial, factores ambientales como la cobertura de bolones, la conductividad, cobertura vegetal y velocidad media de la corriente son determinantes en las estructuración de las comunidades de macroinvertebrados, reportándose tendencias similares para ríos andinos patagónicos argentinos (Miserendino 2001). Un ejemplo, es Hyallela curvispina (Shoemaker 1942), la cual está asociada a sitios con alta conductividad, como ser las estaciones $1,5,7$ y 25 . Además, la vegetación ribereña es un factor estructurador fundamental de las comunidades bentónicas de macroinvertebrados (Lamberty \& Gregory 1996, Flory \& Milner 1999, Leroy \& Marks 2006), influyendo directamente sobre los grupos fragmentadores de hojas y colectores de materia orgánica particulada fina. Por otra parte, durante la época estival, la transparencia del agua y la radiación solar son máximas estimulan la producción primaria favoreciendo de este modo a los grupos de macroinvertebrados que se alimentan de las algas epilíticas (Velásquez \& Miserendino 2003a). Es por esto que en la cuenca del río Baker, se encontró una mayor abundancia de Ephemeroptera, principales consumidores de algas en este tipo de ríos (Díaz-Villanueva \& Modenutti 2004, DíazVillanueva \& Albariño 2003).

En el presente estudio, la variable que mejor explicó los patrones espaciales de los organismos, fue el porcentaje de cobertura de bolones. Este parámetro que se relaciona directamente con otras variables hidráulicas como la velocidad de la corriente y se correlacionó significativamente con la riqueza taxonómica de los macroinvertebrados. Estos resultados reflejan la estabilidad y diversidad de los microhábitats bentónicos del río (Ward 1992), donde existen intersticios que atrapan importantes fracciones de detritos y material vegetal alóctono (Velásquez \& Miserendino $2003 b$ ), que aunque no fue evaluado en el 
presente estudio, deberá ser considerada en investigaciones futuras, con la finalidad de estudiar las variaciones espaciales de la organización funcional de los macroinvertebrados en esta cuenca.

En el caso del sistema fluvial estudiado, la dinámica de los regímenes hidrológicos (e.g. pulsos de inundación) y geomorfológicos (e.g. sedimentación y erosión) está controlada primero, por grandes lagos de origen glacial (e.g. General Carrera) que regulan el caudal y lo mantienen constante a lo largo del año. De los agentes de evacuación de sedimentos, los glaciares limitan sus efectos a los pisos altitudinales superiores, donde construyen morrenas y no participan en la exportación de sedimentos fuera de la montaña. En cambio, los ríos son capaces de exportar grandes cantidades de sedimentos y de organizar una gran cantidad de aspectos funcionales del paisaje fluvial (Ferguson 1984). Las complejas interacciones que se establecen con las variables físicas, dependen fundamentalmente del régimen hidrológico, de los mecanismos de acceso de sedimentos al cauce, las herencias geomorfológicas del Cuaternario Reciente, y de la relación entre producción de sedimentos y capacidad de transporte (Fenn 1987). Consecuentemente, cambios en el hábitat a distintas escalas espaciales, podría tener importantes impactos sobre la distribución de la fauna de macroinvertebrados bentónicos, cuyas consecuencias podrían tener un alcance tanto en tramos de ríos, como hasta la cuenca hidrográfica completa.

\section{AGRADECIMIENTOS}

Se agradece a Marysol Azócar, Cecilia Jiménez, Pablo Pedreros, Alberto Araneda, Oscar Parra, Gustavo Chiang y Héctor Enríquez, por el apoyo brindado en las actividades de terreno y/o laboratorio. También se agradece a la Dirección General de Aguas (DGA) de Región de Aisén, en particular a los señores Francisco Riestra, Jorge O'Kuinghttons y Fabián Espinoza, por su apoyo logístico en terreno. De igual forma se agradece al editor asociado y a los revisores anónimos, cuyas valiosas observaciones permitieron mejorar sustancialmente este artículo, y a todas las personas que directa o indirectamente colaboraron en esta investigación. Este estudio se realizó gracias al financiamiento del proyecto FONDECYT 1070513, con apoyo de los proyectos DIUC/Patagonia 205.310.043$1 \mathrm{sp}$, Complementario del Bicentenario (RUE141) y TWINLATIN Cuenca Baker 018436.

\section{LITERATURA CITADA}

ALBARIÑO R (1997) Spatial distribution of Plecoptera from an Andean-Patagonic lotic environment in Argentina. Revista Brasileira de Biología 57: 629636.

BRAYARD A, G ESCARGUEL \& H BUCHER (2005) Latitudinal gradient of taxonomic richness: Combined outcome of temperature and geographic middomains effects? Journal of Zoological Systematics and Evolutionary 43: 178-188.

BOND G, W BROECKER, S JOHSEN, J MC-MANUS, L LABEYRIE, J JOUZEL \& G BONANI (1993) Correlations between climate records from North Atlantic sediments and Greenland ice. Nature 365: 143-147.

CAMOUSSEIGHT A (2006) Estado de conocimiento de los efemerópteros de Chile. Gayana 70: 50-56.

CAMPOS H, J ARENAS, C JARA, T GONSER \& R PRINS (1984). Macrozoobentos y fauna íctica de las aguas limnéticas de Chiloé y Aysén continentales (Chile). Medio Ambiente 7: 52-64.

CARRASCO J, G CASASSA \& A RIVERA (2002) Meteorological and climatological aspects of the Southern Patagonia Icefield. En: Casassa G, F Sepúlveda \& R Sinclair (eds) The Patagonian Icefields: A unique natural laboratory for environmental and climate change studies: 29-41. Ed. Kluwer Academic/Plenum Publishers.

CASTELLA E, H ADALSTEINSSON, J BRITTAIN, G GISLASON, A LEHMANN et al. (2001) Macrobenthic invertebrate richness and composition along a latitudinal gradient of Europen Glacier-Fed streats. Freshwater Biology 46: 1811 1831 .

CLARKE K, R WARWICK, E SOMER \& R GORLEY (2005) Change in marine communities: An approach to statistical analysis and interpretation. Third edition. Primer, Plymouth. 171pp.

CONAF (1999) Catastro y evaluación de los recursos vegetacionales nativos de Chile. Informe nacional con variables ambientales. Santiago, Chile. 88 pp.

CONNOR E (1986) The role of pleistocene forest refugia in the evolution and biogeograophy of tropical biotas. Trends in Ecology and Evolution 1: 165168.

CRUCES P, J CERDA \& M AHUMADA (1999) Levantamiento para el ordenamiento de los ecosistemas de Aysén. Servicio Agrícola Ganadero, Santiago, Chile. 137 pp.

DGA (2005) Propuesta de norma secundaria de calidad ambiental para la protección de las aguas de la cuenca del río Aysén. Dirección General de Aguas, Chile. 15 pp.

DÍAZ-VILLANUEVA V \& R ALBARIÑO (2003) Algal ingestion and digestion by two ephemeropteran larvae from a Patagonian Andean stream. Proceedings of the $14^{\text {th }}$ International Symposium on Ephemeroptera: 384-391. 
DÍAZ-VILLANUEVA V \& B MODENUTTI (2004) Experimental analysis of grazing by the mayfly Meridialaris chiloeensis on different successional stages of stream periphyton. International Review of Hydrobiology 89: 263-277.

DOISY KE \& CF RABENI (2001) Flow conditions, benthic food resources, and invertebrate community composition in a low-gradient stream in Missouri. Journal of the North American Benthological Society 20: 17-32.

DOMÍNGUEZ E, O MOLINERI, M PESCADOR, M HUBBARD \& C NIETO (2006) Ephemeroptera of South America. En: Adis J, R Arias, G RuedaDelgado \& KM Wantzen (eds) Aquatic biodiversity in Latin America. Vol 2. Pensoft. Moscow and Sofia. $646 \mathrm{pp}$

FERGUSON R (1984) Magnitude and modelling of snowmelt runoff in the Cairngorm Mountains, Scotland. Hydrological Sciences Journal 29: 49-62.

FERNÁNDEZ HR \& E DOMÍNGUEZ (2001) Guía para la determinación de los artrópodos bentónicos sudamericanos. EUDET. Tucumán, Argentina. 450 $\mathrm{pp}$

FENN C (1987) Sediment transfer processes in Alpine glacier basins. En: Gurnell AM \& MJ Clark (eds) Glacio fluvial sediment transfer: An alpine perspective: 59-86. Wiley, Chichester.

FOLK R (1980) Petrology of sedimentary rocks. Hemphill Publishing, Austin. 184 pp.

FIELD J, K CLARKE \& R WARWICK (1982) A practical strategy for analyzing multispecies distribution patterns. Marine Ecology Progress Series 8: 37-52.

FLORY E \& AM MILNER (1999) Influence of riparian vegetation on invertebrate assemblages in a recent formed stream in Glacier Bay National Park, Alaska. Journal of the North American Benthological Society 18: 261-273.

FUENZALIDA H (1965) Biogeografía. En: Geografía Económica de Chile: 228-267. Texto Refundido. Corporación de Fomento de la Producción. Santiago, Chile.

GARCÍA PE \& DA AÑÓN-SUÁREZ (2007) Community structure and phenology of Chironomids (Insecta: Chironomidae) in a Patagonian Andean stream. Limnologica 37: 109-117.

GONZÁLEZ E (2003) The freshwater amphipods Hyalella Smith, 1874 in Chile (Crustacea: Amphipoda). Revista Chilena de Historia Natural 76: 623-637.

HABIT E, B DYER \& I VILA (2006) Estado de conocimiento de los peces dulceacuícolas de Chile. Gayana 70: 16-23.

HAUENSTEIN E (2006) Estado de conocimiento de las macrófitas dulceacuícolas de Chile. Gayana 70: 1623.

HECKMAN C (2003) Encyclopedia of South American aquatic insects: Plecoptera. Kluwer Academic Publishers, Netherlands. 329 pp.

INSTITUTO GEOGRÁFICO MILITAR (1984) Geografía de Chile. En: Hidrología. Primera edición. Santiago, Chile. 320 pp

HEWITT G (2000) The genetic legacy of the Quaternary ice ages. Nature 405: 907-913.

HEWITT G (1996) Some genetic consequences of ice ages, and their role in divergence and speciation. Biological Journal of the Linnean Society 58: 247 276.

HULINGS NC \& JS GRAY (1971) A manual for the study of meiofauna. Smithsonian Contributions to Zoology 78: 1-84.

JARA C, E RUDOLPH \& E GONZÁLEZ (2006) Estado de conocimiento de los crustáceos malacostracos dulceacuícolas de Chile. Gayana 70: 72-81.

JEREZ V \& E MORONI (2006) Estado de conocimiento de los coleópteros dulceacuícolas de Chile. Gayana 70: $72-81$

JOWETT I (2003) Hydraulic constraints on habitat suitability for benthic invertebrates in gravel-bed rivers. River Research and Applications 19: 495507.

LAMBERTY GA \& SV GREGORY (1996) Transport and retention of CPOM. En: Hauer FR \& GA Lamberti (eds) Methods in stream ecology: 217-229. Academic Press, San Diego, California

LEROY C \& J MARKS (2006) Litter quality, stream characteristics and litter diversity influence decomposition rates and macroinvertebrates. Freshwater Biology 51: 605-617.

LOPRETTO EC \& G TELL (1995) Ecosistemas de aguas continentales. Hemisferio Sur, La Plata. 1401 pp.

LUGO-ORTIZ C \& W McCAFFERTY (1998) Five new genera of Baetidae (Insecta: Ephemeroptera) from South America. Annales des Limnology 34: 57-73.

MARTÍNEZ-HARMS M \& R GAJARDO (2008) Ecosystem value in the Western Patagonia protected areas. Journal for Nature Conservation 16: 72-87.

MCLELLAN I, MISERENDINO ML \& MET HOLLMANNN (2006) Two new species of Notoperla (Plecoptera: Gripopterygidae) and a redescription of Notoperlopsis femina. Zootaxa 1140: 53-68.

MCLELLAN I, M MERCADO \& S ELLIOTT (2005) A new species of Notoperla (Plecoptera: Gripopterygidae) from Chile. Illiesia 1:33-39.

MILANO D, V CUSSAC, P MACCHI, D RUZZANTE, MF ALONSO, PH VIGLIANO \& MA DENEGRI (2002) Predator associated morphology in Galaxias platei in Patagonian lakes. Journal of Fish Biology 61: 138-156.

MISERENDINO L (2006) Seasonal and spatial distribution of stoneflies in the Chubut River (Patagonia, Argentina). Hydrobiologia 568: 263274.

MISERENDINO ML (1995) Composición y distribución del macrozoobentos de un sistema lotico andinopatagónico. Ecología Austral 5: 133-142.

MISERENDINO ML \& LA PIZZOLÓN (2000) Macroinvertebrates of a fluvial system in Patagonia: Altitudinal zonation and functional structure. Archiv für Hydrobiologie 150: 55-83.

MISERENDINO M (2001) Macroinvertebrate assemblages in Andean Patagonian rivers and streams: Environmental relationships. Hydrobiologia 444: 147-158.

MISERENDINO M \& L PIZZOLON (2004) Interactive effects of basin features and land-use change on macroinvertebrates communities of headwater streams in the Patagonian Andes. River Research and Applications 20: 967-983.

NARUSE R \& M ANIYA (1995) Synopsis of glacier researches in Patagonia. Bulletin of Glaciers Research 13: 1-10.

NIEMEYER H, J SKÁRMETA, R FUENZALIDA \& W ESPINOSA (1984) Hoja Península de Taitao y Puerto Aisén, Región de Aisén del General Carlos Ibáñez del Campo. Servicio Nacional de Geología y Minería, Carta Geológica de Chile: 60-61.

OLMOS V \& G MUÑOZ (2006) Estado de conocimiento de los parásitos metazoos de organismos dulceacuícolas de Chile. Gayana 70: 122-139. 
ORELLANA M (2006) Estado de conocimiento de los briozoos dulceacuícolas de Chile. Gayana 70: 9699.

ORTIZ J (2006) Estado de conocimiento de los anfibios de Chile. Gayana 70: 114-121.

OYANEDEL A, C VALDOVINOS, C MOYA, M AZÓCAR, G MANCILLA \& R FIGUEROA (2008) Patrones de distribución espacial de los macroinvertebrados bentónicos de la cuenca del río Aisén. Gayana 72: 241-257.

PARADA E \& S PEREDO (2006) Estado de conocimiento de los bivalvos dulceacuícolas de Chile. Gayana 70: 82-87.

PARRA O (2006) Estado de conocimiento de las microalgas dulceacuícolas de Chile (excepto diatomeas). Gayana 70: 8-15.

PESSACQ P \& ML MISERENDINO (2008) Ephemeroptera and Plecoptera biodiversity in central Patagonia, Chubut province, Argentina. Zootaxa 1817: 27-38

RIVERA P (2006) Estado de conocimiento de las diatomeas dulceacuícolas de Chile. Gayana 70: 1-7.

RODRÍGUEZ R, A MARTICORENA \& E TENEB (2008) Plantas vasculares de los ríos Baker y Pascua, Región de Aisén, Chile. Gayana Botánica 65: 3970.

ROJAS F (2006) Estado de conocimiento de los tricópteros de Chile. Gayana 70: 65-71.

ROMERO VF (2001) Guía para la determinación de artrópodos bentónicos sudamericanos. En: Fernández HR \& E Domínguez (eds) Plecoptera: 93-109. Serie Investigaciones de la UNT. Subserie Ciencias Exactas y Naturales, Universidad Nacional de Tucumán, Tucumán, Argentina

ROSENBLÜTH B, H FUENZALIDA \& P ACEITUNO (1998) Recent temperature variations in Southern South America. International Journal of Climatology 17: 67-85.

SGANGA JV \& MS FONTANARROSA (2006). Contribution to the knowledge of the preimaginal stages of the genus Smicridea McLachlan in South America (Trichoptera: Hydropsychidae: Smicrideinae). Zootaxa 1258: 1-15.

STATZNER B \& T HOLM (1982) Morphological adaptations of benthic invertebrates to stream flow an old question studied by means of new technique (Laser Doppler Anemometry). Oecologia 53: 290292.

TABERLET P \& R CHEDDADI (2002) Quaternary refugia and persistence of biodiversity. Science 297: 2009-2010.

VALDOVINOS C (2006) Estado de conocimiento de los gastrópodos dulceacuícolas de Chile. Gayana 70: $88-95$.

VELÁSQUEZ SM \& ML MISERENDINO (2003a) Análisis de la materia orgánica alóctona y organización funcional de macroinvertebrados en relación con el tipo de hábitat en ríos de montaña de Patagonia. Ecología Austral 13: 67-82.

VELÁSQUEZ SM \& ML MISERENDINO (2003b) Habitat type and macroinvertebrate assemblages in low order Patagonian streams. Archiv fur Hydrobiologie 158: 461-483

VERA A \& A CAMOUSSEIGHT (2006) Estado de conocimiento de los plecópteros de Chile. Gayana 70: 57-64.

VICTORIANO P, A GONZÁLEZ \& R SCHLATTER (2006) Estado de conocimiento de las aves de ecosistemas dulceacuícolas de Chile. Gayana 70: 140-162.

VILA I, L FUENTES \& M CONTRERAS (1999) Peces Límnicos de Chile. Boletín Museo Historia Natural (Chile) 48: 61-75.

VILLALOBOS C, J ORTIZ-SANDOVAL \& E HABIT (2008) Finding of Gordius austrinus de Villalobos, Zanca \& Ibarra - Vidal, 2005 (Gordiida, Nematomorpha) in the stomach of Salmo trutta (Salmoniformes) in Patagonia. Gayana 72: 31-35.

VILLALOBOS L (2006) Estado de conocimiento de los crustáceos zooplanctónicos dulceacuícolas de Chile. Gayana 70: 31-39

WAIS I (1987) Macrozoobenthos of Negro River Basin, Argentine, Patagonia. Studies on Neotropical Fauna \& Environment 22: 73-91.

WAIS I (1990) A checklist of the benthic macroinvertebrates of the Negro River Basin, Patagonia, Argentina, including an approach to their functional feeding groups. Acta Limnologica Brasiliensia 3: 829-845.

WAIS I \& A BONNETO (1988) Analysis of the allochthonous organic matter and associated macroinvertebrates in some streams of Patagonia (Argentina). Verhandlungen der Internationale Vereinigung fur Theoretische und Angewandte der Limnologie 23: 1455-1459.

WARD JV (ed) (1992) Aquatic insect ecology 1, biology and habitat. John Wiley \& Sons, Inc, New York. $384 \mathrm{pp}$.

WOELFL S (2006) Estado de conocimiento de los protozoos planctónicos dulceacuícolas de Chile. Gayana 70: 24-26.

ZAPATA J (2006) Estado de conocimiento de los tecamebianos dulceacuícolas de Chile. Gayana 70: 27-30. 